\title{
A laserterapia de baixa potência melhora o desempenho muscular mensurado por dinamometria isocinética em humanos
}

\author{
Low-level laser therapy enhances muscular performance as measured by isokinetic \\ dynamometry in humans
}

\author{
Ernesto Cesar Pinto Leal Junior ${ }^{1}$, Francis Régio Nassar², Shaiane da Silva Tomazoni ${ }^{3}$, \\ Jan Magnus Bjordal', Rodrigo Álvaro Brandão Lopes-Martins ${ }^{5}$
}

Estudo desenvolvido no Depto. de Fisioterapia da Univap - Universidade do Vale do Paraíba, São José dos Campos, SP, Brasil

1 Prof. Dr. do Programa de PósGraduação em Ciências da Reabilitação da Universidade Nove de Julho, São Paulo

2 Prof. Ms. do Curso de Fisioterapia da Universidade Paulista, São José dos Campos

3 Fisioterapeuta; mestranda no Laboratório de Farmacologia e Terapêutica Experimental do ICB/USP - Instituto de Ciências Biomédicas da Universidade de São Paulo, São Paulo

4 Prof. Dr. do Centro de Prática Baseada em Evidência, Faculdade de Ciências Sociais e da Saúde, Universdade de Bergen, Bergen, Noruega

5 Prof. Dr. do ICB/USP

ENDEREÇO PARA CORRESPONDÊNCIA:

Ernesto Cesar P. Leal Jr. PPG Ciências da Reabilitação / Uninove

R. Vergueiro 325 01504-001

São Paulo SP

e-mail: emesto.leal.junior@gmail.com

\section{APRESENTAÇÃO}

nov. 2009

ACEITO PARA PUBLICAÇÃO ago. 2010
Resumo: A fadiga muscular é uma nova área de pesquisa em laserterapia, com poucos estudos conduzidos. Embora a laserterapia de baixa potência (LBP) previamente ao exercício tenha apresentado resultados positivos no retardo da fadiga musculoesquelética, ainda não foi estudada utilizando-se a dinamometria isocinética para mensurar desempenho e fadiga muscular. Este estudo tem o objetivo de avaliar os efeitos da LBP $(655 \mathrm{~nm}, 50 \mathrm{~mW}, 2,4$ J por ponto e $12 \mathrm{~J}$ de energia total) sobre o desempenho e fadiga muscular do músculo tibial anterior, utilizando dinamometria isocinética (30 repetições de contração concêntrica) em 14 indivíduos saudáveis sedentários do sexo masculino. Os voluntários foram avaliados ao efetuar 30 repetições isocinéticas de dorsiflexão de tornozelo à velocidade angular de $240^{\circ}$. $\mathrm{seg}^{-1}$. Os resultados mostram que, quando os voluntários foram tratados com LBP antes do exercício, os valores do pico de torque $(30,91 \pm 5,86$ N.m) foram significativamente superiores, comparados a três medições anteriores sem a aplicação de $\operatorname{LBP}(24,92 \pm 7,45$ N.m, $p<0,001 ; 26,83 \pm 7,74$ N.m, $p<0,01$;

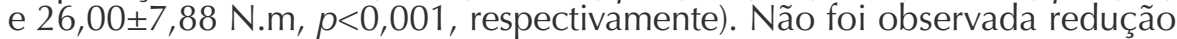
no índice de fadiga. Conclui-se que a LBP aumenta o torque gerado pelos músculos irradiados, melhorando assim o desempenho musculoesquelético, porém sem interferir no índice de fadiga.

Descritores: Fadiga muscular; Dinamômetro de força muscular; Homens; Terapia a laser de baixa intensidade

Abstract: Skeletal muscle fatigue is a novel research area in laser therapy, there being few studies carried out. Though low-level laser therapy (LLLT) applied prior to exercise has showed positive results in delaying skeletal muscle fatigue, no studies could be found that measured muscle performance and fatigue by means of isokinetic dynamometry. This clinical trial aims at assessing the effects of LLLT $(655 \mathrm{~nm}, 50 \mathrm{~mW}$ and $12 \mathrm{~J}$ total energy delivered) on anterior tibialis muscle performance and fatigue by means of isokinetic dynamometry (30 concentric-mode repetitions at $240^{\circ} . \mathrm{sec}^{-1}$ angular speed) in 14 healthy male subjects. Results show that, when volunteers had been treated with LLLT prior to exercise, torque peak values $(30.91 \pm 5.86 \mathrm{~N} . \mathrm{m})$ were significantly higher than those of three previous measurements with no LLLT $(24.92 \pm 7.45$ N.m, $p<0.001 ; 26.83 \pm 7.74$ N.m, $p<0.01 ;$ and $26.00 \pm 7.88$ N.m, $p<0.001)$. However, no decrease in fatigue indexes could be found. It may thus be said that LLLT increased skeletal muscle torque in irradiated muscles, but had no effect on muscle fatigue.

Key words: Muscle fatigue; Laser therapy, low-level; Men; Muscle strength dynamometer 


\section{INTRODUÇÃO}

Os mecanismos de ação, desenvolvimento e prevenção da fadiga muscular não são totalmente elucidados. São comumente observados como características de fadiga musculoesquelética a diminuição da força, comprometimento do controle motor e dor muscular subseqüente 1 .

A fadiga muscular pode ser dividida em um componente central e um componente periférico. O tipo e intensidade do exercício, grupos musculares envolvidos o ambiente físico e bioquímico são fatores periféricos que afetam a produção de força durante a atividade muscular. Dessa forma, a fadiga muscular é um processo complexo que envolve elementos fisiológicos, biomecânicos e psicológicos 2 . Idade e sexo também são fatores importantes, pois determinam a habilidade de contração musculoesquelética para tolerar a fadiga ${ }^{3}$. Existem também indícios de que a força exercida declina mais rapidamente em indivíduos do sexo masculino, durante o desenvolvimento da fadiga esquelética 4 .

Há vários tipos de fadiga muscular e a contribuição de cada um para a diminuição geral no desempenho muscular depende do tipo de fibra muscular, da intensidade e da duração da atividade ${ }^{5}$. Um dos tipos é causado pelo acúmulo de $\mathrm{K}^{+}$no sistema tubular transverso das fibras musculares. Outro é a fadiga metabólica, devida a efeitos diretos ou indiretos do acúmulo de metabólitos como fosfato inorgânico, adenosina difosfato, espécies reativas de oxigênio (ROS) e diminuição dos substratos (adenosina trifosfato - ATP, creatino-fosfato e glicogênio) ${ }^{6}$. A diminuição na geração de força em condições anaeróbias, como exercícios extenuantes, inevitavelmente gera espécies reativas de oxigênio e piora a função mitocondrial que, por sua vez, causa despolarização das células musculares, responsável pela diminuição na capacidade de gerar força7.

A laserterapia de baixa potência (LBP) sobre a fadiga musculoesquelética é uma nova área de pesquisa cujos parâmetros ideais de aplicação não são completamente conhecidos. Em um experimento prévio com animais, descobrimos que a LBP poderia retardar o inevitável declínio da contração máxima durante repetidas contrações tetânicas induzidas eletricamente ${ }^{8}$. Doses específicas de LBP reduziram os níveis da atividade de creatino-quinase muscular, em comparação com o grupo que não recebeu irradiação, indicando portanto uma diminuição do dano muscular. Posteriormente, foi conduzido um estudo clínico com 12 atletas profissionais de voleibol utilizando laser com o mesmo comprimento de onda do estudo com animais (655 nm), com $50 \mathrm{~mW}$ de potência de saída e $5 \mathrm{~J}$ aplicados em cada um dos quatro pontos igualmente distribuídos ao longo do ventre muscular do bíceps braquial (20 J de energia total aplicada); observou-se um aumento significativo no número de contrações executadas e no tempo utilizado para a realização das contrações, com um pequeno aumento nos níveis de lactato sangüíneo ${ }^{9}$. Em um terceiro estudo10, um ensaio clínico randomizado cruzado, foi utilizado o mesmo protocolo e mensurados os mesmos parâmetros do estudo anterior, para avaliar os efeitos da LBP com um comprimento de onda infravermelho $(830 \mathrm{~nm}, 100 \mathrm{~mW}$ e 5 J em cada um dos quatro pontos ao longo do ventre muscular do bíceps braquial, $20 \mathrm{~J}$ de energia total) sobre a fadiga muscular do bíceps braquial. Nesse estudo, a aplicação de LBP aumentou significativamente o número de repetições realizadas por dez atletas profissionais de voleibol, porém sem diferenças significantes no tempo utilizado para realizar as contrações. Além disso, não houve diferença significante nos níveis de lactato sanguíneo pós-exercício entre o tratamento com LBP e placebo 10 .

No entanto, nos dois ensaios clínicos citados acima, embora apresentem direta aplicabilidade clínica, não foi empregado o padrão ouro na mensuração de fadiga e desempenho muscular. Atualmente a dinamometria isocinética é considerada a melhor ferramenta para medir o desempenho musculoesquelético e, conseqüentemente, a fadiga 11 . Por isso, este estudo visa mensurar os efeitos da LBP com o comprimento de onda de $655 \mathrm{~nm}$ sobre o desempenho e fadiga musculoesquelética, utilizando como instrumento de medida a dinamometria isocinética.

\section{METODOLOGIA}

Este estudo foi aprovado pelo Comitê de Ética em Pesquisa da Universidade do Vale do Paraíba. Participaram do estudo 14 indivíduos saudáveis do sexo masculino, que assinaram um termo de consentimento livre e esclarecido. Foram incluídos no estudo indivíduos saudáveis do sexo masculino, com idade entre 18 e 25 anos, não-praticantes de atividade física regular. Foram excluídos os sujeitos que utilizassem medicamentos e/ou suplementos nutricionais, fossem praticantes de atividade física regular ou que, no decorrer do estudo, passassem a ter prática regular de atividade física, além dos sujeitos que sofreram lesão musculoesquelética nos membros inferiores nos dois meses prévios à realização do estudo ou que, no decorrer do estudo, sofressem lesão musculoesquelética nos membros inferiores.

\section{Procedimentos}

O estudo foi dividido em quatro fases com um intervalo de 72 horas entre cada uma, para propiciar adequada recuperação muscular dos voluntários. Tendo em vista que o exercício (ou contração) isocinético não é uma atividade habitual, optou-se por realizar três fases idênticas (fases 1, 2 e 3) com o objetivo de familiarizar os voluntários ao protocolo de exercício. Os voluntários foram instruídos a não mudar seus hábitos alimentares e atividades físicas durante o período total do estudo.

Ns fases 1 e 2, chamadas de adaptação, cada voluntário efetuou 30 repetições isocinéticas de dorsiflexão de tornozelo à velocidade angular de $240^{\circ} . \mathrm{seg}^{-1}$. Nenhum tratamento foi aplicado previamente ao protocolo. $\mathrm{Na}$ terceira fase, considerada controle, os participantes novamente realizaram 30 repetições isocinéticas de dorsiflexão, à mesma velocidade, também sem tratamento prévio.

Na fase 4 (LBP), novamente efetuaram as mesmas 30 repetições isoci- 
néticas de dorsiflexão de tornozelo à velocidade angular de $240^{\circ}$. seg $^{-1}$, porém, cinco minutos antes de realizarem o protocolo, receberam tratamento com LBP em cinco pontos ao longo do ventre muscular do tibial anterior (Figura 1).

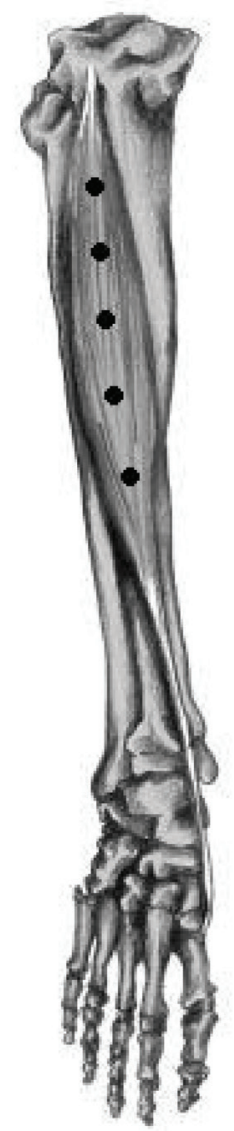

Figura 1 Pontos de aplicação da LBP no músculo tibial anterior (círculos pretos)
Antes de cada avaliação por meio de dinamometria isocinética (nas quatro fases), os indivíduos efetuaram séries de 30 segundos de alongamento ativo dos músculos tibial anterior e tríceps sural do membro inferior dominante.

Para a avaliação isocinética, os voluntários foram sentados em ângulo de $100^{\circ}$ entre o tronco e o quadril, com a perna não-dominante posicionada a $90^{\circ}$ de flexão de joelho e fixada ao assento do dinamômetro por um cinto; a perna dominante foi posicionada a $30^{\circ}$ de flexão de quadril e também foi fixada ao assento com um cinto. Os voluntários também foram presos ao assento por dois cintos cruzando seu tronco e, durante o teste, foram instruídos a posicionar os braços cruzando o tronco. O eixo do dinamômetro isocinético foi posicionado paralelamente ao centro da articulação do tornozelo. Nos testes, os dados foram colhidos por um dinamômetro isocinético Biodex (System 3, Shirley, NY, USA) no modo concêntrico; durante todo o teste os indivíduos receberam estímulos verbais do examinador para efetuar as contrações.

O tratamento com LBP, como dito, foi aplicado apenas na fase 4, após o alongamento muscular. Após a irradiação, os indivíduos foram posicionados sentados no assento do dinamômetro. $\mathrm{O}$ protocolo foi iniciado 5 minutos depois da irradiação da LBP no último ponto de tratamento do músculo tibial anterior. Os parâmetros utilizados para a LBP estão expostos no Quadro 1.
Quadro 1 Parâmetros utilizados na aplicação da LBP

\begin{tabular}{|c|c|}
\hline Comprimento de onda & 655 nm (vermelho) \\
\hline Frequência & modo contínuo \\
\hline Potência de saída & $50 \mathrm{~mW}$ \\
\hline Diâmetro do spot & $0,06 \mathrm{~cm}$ \\
\hline Área do spot & $0,01 \mathrm{~cm}^{2}$ \\
\hline Densidade de potência & $5 \mathrm{~W} / \mathrm{cm}^{2}$ \\
\hline Energia irradiada por ponto & 2,4 Joules por ponto \\
\hline Densidade de energia & $240 \mathrm{~J} / \mathrm{cm}^{2}$ por ponto \\
\hline Tempo de irradiação & 48 segundos por ponto \\
\hline \multicolumn{2}{|c|}{ № de pontos irradiados por músculo cinco } \\
\hline Energia total irradiada & 12 Joules \\
\hline \begin{tabular}{l|l} 
Modo de & Probe estacionári \\
aplicação & a pele, em ângulc
\end{tabular} & $\begin{array}{l}\text { io em contato direto com } \\
\text { o de } 90^{\circ} \text { e leve pressão }\end{array}$ \\
\hline
\end{tabular}

\section{Análise estatística}

Os valores médios e os respectivos desvios padrões foram utilizados para análise estatística. Foi utilizado o teste Anova, com pós-teste de Tukey-Kramer, para testar se houve diferença estatisticamente significante nos resultados de índice de fadiga (declínio do trabalho muscular realizado no último terço do protocolo de fadiga pelo trabalho realizado no primeiro terço - em valores percentuais) e pico de torque (valor máximo de torque obtido durante a realização do protocolo de fadiga) entre as quatro fases do estudo (adaptação 1, adaptação 2, controle e LBP). O nível de significância foi de $p<0,05$.

\section{RESULTADOS}

Catorze homens sedentários saudáveis foram recrutados para o estudo, com idade média de 23,07 $\pm 1,94$ anos, massa corporal de $76,36 \pm 5,93 \mathrm{~kg}$ e estatura média de 178,14 $\pm 5,11 \mathrm{~cm}$.

$O$ índice de fadiga mensurado por dinamometria isocinética foi de $37,95 \pm 7,04 \%$ na fase de adaptação $1,37,70 \pm 6,60 \%$ na fase de adaptação $2,41,94 \pm 7,64 \%$ na fase controle e $38,07 \pm 6,77 \%$ na fase LBP, sem diferença estatística $(p<0,05)$ entre as quatro fases. Os resultados do índice de fadiga são ilustrados na Figura 2.

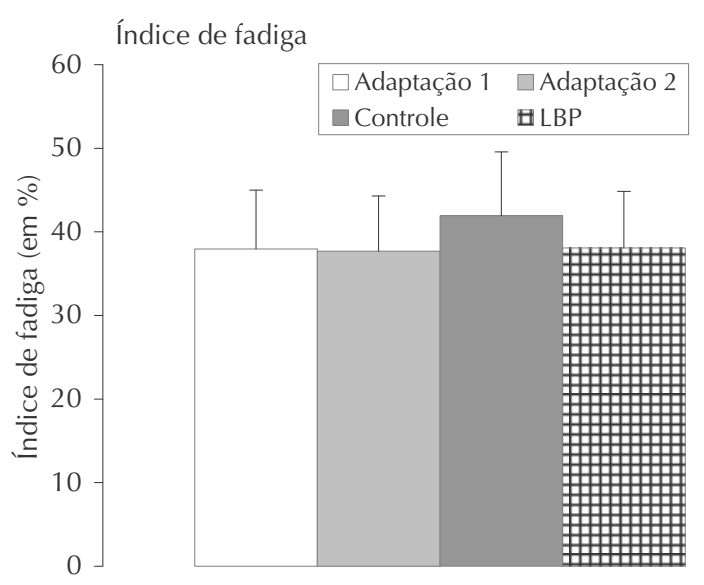

Figura 2 Índice de fadiga nas quatro fases do estudo
O pico de torque mensurado foi de 24,92 $\pm 7,45$ N.m na fase de adaptação 1 , $26,83 \pm 7,74$ N.m na adapta-

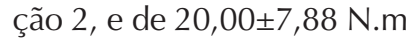
na fase controle, também sem diferenças estatisticamente significantes entre essas três fases $(p>0,05)$. O pico de torque na fase LBP foi de $30,91 \pm 5,86$ N.m, foi significativamente superior às fase de adaptação $1(p<0,001)$, de adaptação $2(p<0,01)$ e controle $(p<0,001)$. Os resultados do pico de torque são ilustrados na Figura 3. 


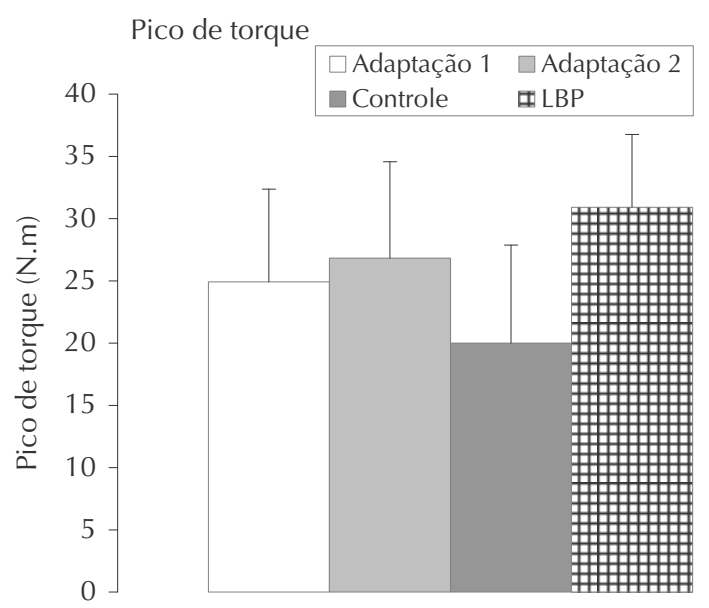

Figura 3 Pico de torque nas quatro fases do estudo

\section{DISCUSSÃO}

No presente estudo observou-se um aumento significativo no pico de torque executado pelos voluntários quando os receberam tratamento com LBP antes da avaliação por dinamometria isocinética. Resultados similares foram observados em um estudo pré-clínico de Lopes-Martins et al. ${ }^{8}$, em que se utilizaram contrações tetânicas para induzir fadiga musculoesquelética no músculo tibial anterior de ratos. Quando a LBP foi aplicada previamente às contrações tetânicas, foi observado que a força de contração aumentou significativamente, fato similar ao encontrado no presente estudo.

Como mencionado na Introdução, ensaios clínicos recentes apresentaram resultados positivos a favor da LBP no retardo da fadiga musculoesquelética. Leal Jr et al. ${ }^{9}$ utilizaram o comprimento de onda vermelho de $655 \mathrm{~nm}$ em atletas profissionais de voleibol masculino; o número de contrações realizadas e o tempo para executar essas contrações sofreram aumento significativo nos atletas que receberam tratamento com LBP previamente ao exercício. Os mesmos autores, em outro estudo 10 com o mesmo protocolo para induzir fadiga musculoesquelética, testaram os efeitos do comprimento de onda infravermelho de $830 \mathrm{~nm}$, observando novamente que o número de contrações realizadas pelos atletas profissionais de voleibol aumentou significativamente, mas que o tempo para efetuar as contrações não sofreu alteração. Os dados de ambos os estudos indicam que os vo- luntários foram capazes de realizar um número maior de contrações musculares quando tratados previamente com LBP, aumentando conseqüentemente $\mathrm{O}$ trabalho muscular realizado e indicando que a fadiga muscular foi atenuada.

A dinamometria isocinética foi previamente utilizada para mensurar desempenho e fadiga musculoesquelética no estudo de Gorgey et al. 12 , que induziram a fadiga por um estímulo elétrico no músculo quadríceps de cinco voluntários saudáveis e aplicaram a LBP com um laser de varredura (scanner) de 808 nm. Os autores não observaram diferença no desempenho e fadiga muscular comparando a situação do grupo controle com as situações LBP utilizando 3 J e 7 J. No entanto, alguns fatores como a utilização de estimulação elétrica (modalidade passiva) para induzir a fadiga e a utilização de um laser scanner (não promovendo um tempo suficiente de interação do laser com o tecido) em não-contato com a pele (diminuindo possivelmente a penetração da irradiação), podem ser considerados limitações do estudo e provavelmente influenciaram negativamente os resultados. A partir da observação desses fatores foi que, no presente estudo, decidiu-se utilizar exercício ativo para induzir a fadiga musculoesquelética e, para a irradiação, um probe estacionário (aumentando o tempo de interação laser-tecido) em contato com a pele, aumentando a penetração da LBP.

Não foram encontradas diferenças entre as duas fases de adaptação e a fase controle, o que indica que os valores de pico de torque obtidos na fase LBP foram ocasionados pelos efeitos da irradiação, e não por outros fatores, como adaptação dos indivíduos ao protocolo.

Entretanto, não foram observadas diferenças quanto ao índice de fadiga nas quatro fases do estudo. Isso pode ter ocorrido devido à opção pelo uso do comprimento de onda vermelho. Sabe-se que tais comprimentos de onda (na faixa do vermelho) têm menor penetração na pele quando comparados a comprimentos de onda infravermeIhos 13 , o que pode ter levado a uma menor quantidade de energia sendo entregue ao tecido alvo (tecido muscular), ocasionando efeitos apenas nas primeiras contrações realizadas durante a execução do protocolo; os efeitos positivos se sentiram apenas no pico de torque, que usualmente é alcançado no primeiro terço do exercício.

Os mecanismos da LBP para aumentar o desempenho musculoesquelético não são completamente conhecidos, porém alguns efeitos da LBP sobre o estresse oxidativo, a atividade mitocondrial e microcirculação são bem conhecidos, podendo assim explicar as maneiras pelas quais o desempenho musculoesquelético é aumentado pela LBP.

Estudos feitos com animais indicam que, por mecanismos locais, a LBP pode prevenir lesões musculares isquêmicas ao reduzir a atividade de creatino-fosfoquinase e a liberação de espécies reativas de oxigênio (ROS), enquanto aumenta os níveis de antioxidantes e proteínas de choque térmico14,15. Esses resultados são interessantes, uma vez que os resultados descritos por Ortenblad e Stephenson 16 mostraram que um músculo exercitado intensamente sob condições anaeróbicas iria gerar grandes quantidades de ROS, levando a sua despolarização e reduzindo sua força. Dessa forma, a LBP pode ser útil para aumentar o desempenho muscular. Em um estudo recente, de $\mathrm{Xu}$ et al.17, a LBP aumentou a função mitocondrial em células musculares sob doses de $0,33-8,22 \mathrm{~J} / \mathrm{cm}^{2}$; e doses de LBP de 0,33 e $1,338 \mathrm{~J} / \mathrm{cm}^{2}$ reverteram o estado de disfunção mitocondrial induzida pela estimulação elétrica.

Outro estudo recente 18 com LBP AsGa 904 nm demonstrou que a dosagem de $5 \mathrm{~J} / \mathrm{cm}^{2}$, aplicada após uma lesão traumática no músculo gastrocnêmio em ratos, pode aumentar a atividade de cadeias respiratórias mitocondriais do complexo I, II, III e IV, e de succinato desidrogenase durante o processo de 
cicatrização muscular. Esses resultados sugerem que a LBP pode aumentar a síntese de ATP, fato que pode estar diretamente relacionado com o aumento do desempenho muscular observado no presente estudo.
Por se tratar de uma nova área de pesquisa, são necessários mais estudos para verificar os efeitos da LBP no retardo da fadiga muscular, bem como para definir uma "janela terapêutica" para a aplicação da LBP com essa finalidade.
Conclui-se que a LBP, aplicada com os parâmetros utilizados neste estudo, melhora o desempenho do músculo tibial anterior, sem interferir no desenvolvimento da fadiga muscular.

\section{REFERÊNCIAS}

1 Green S, Langberg H, Skovgaard D, Bulow J, Kjaer M. Interstitial and arterial-venous $\left[\mathrm{K}^{+}\right]$in human calf muscle during dynamic exercise: effect of ischaemia and relation to muscle pain. J Physiol. 2000;529(4 Pt 3):849-61.

2 Weir JP, Beck TW, Cramer JT, Housh TJ. Is fatigue all in your head? A critical review of the central governor model. Br J Sports Med. 2006;40(7):573-86.

3 Hurley BF. Age, gender, and muscular strength. J Gerontol A Biol Sci Med Sci. 1995;50(Spec no):41-4.

4 Szubski C, Burtscher M, Loscher WN. Neuromuscular fatigue during sustained contractions performed in shortterm hypoxia. Med Sci Sports Exerc. 2007;39(6):948-54.

5 Billaut F, Basset FA, Giacomoni M, Lemaitre F, Tricot V, Falgairette G. Effect of high-intensity intermittent cycling sprints on neuromuscular activity. Int J Sports Med. 2006;27(1):25-30.

6 Allen DG, Lamb GD, Westerblad H. Skeletal muscle fatigue: cellular mechanisms. Physiol Rev. 2008;88(1):287-332.

7 Nethery D, Callahan LA, Stofan D, Mattera R, DiMarco A, Supinski G. PLA(2) dependence of diaphragm mitochondrial formation of reactive oxygen species. J Appl Physiol. 2000;89(1):72-80.

8 Lopes-Martins RA, Marcos RL, Leonardo PS, Prianti Jr AC, Muscara MN, Aimbire F, et al. Effect of low-level laser (Ga-Al-As $655 \mathrm{~nm}$ ) on skeletal muscle fatigue induced by electrical stimulation in rats. J Appl Physiol. 2006;101(1):283-8.

9 Leal Junior EC, Lopes-Martins RA, Dalan F, Ferrari M, Sbabo FM, Generosi RA, et al. Effect of 655-nm low level laser therapy on exercise-induced skeletal muscle fatigue in humans. Photomed Laser Surg. 2008;26(5):419-24.
10 Leal Junior EC, Lopes-Martins RA, Vanin AA, Baroni BM, Grosselli D, De Marchi T, et al. Effect of 830-nm lowlevel laser therapy on exercise-induced skeletal muscle fatigue in humans. Lasers Med Sci. 2009;24(3):425-31.

11 Brown L. Isokinetics in human performance. Champaign: Human Kinetics; 2000.

12 Gorgey AS, Wadee AN, Sobhi NN. The effect of low-level laser therapy on electrically induced muscle fatigue: a pilot study. Photomed Laser Surg. 2008;26(5):501-6.

13 Enwemeka CS. Intricacies of dose in laser phototherapy for tissue repair and pain relief. Photomed Laser Surg. 2009;27(3):387-93.

14 Avni D, Levkovitz S, Maltz L, Oron U. Protection of skeletal muscles from ischemic injury: low-level laser therapy increases antioxidant activity. Photomed Laser Surg. 2005;23(3):273-7.

15 Rizzi CF, Mauriz JL, Correa DSF, Moreira AJ, Zettler CG, Filippin LI, et al. Effects of low-level laser therapy (LLLT) on the nuclear factor (NF)-kappaB signaling pathway in traumatized muscle. Lasers Surg Med. 2006;38(7):704-13.

16 Ortenblad N, Stephenson DG. A novel signaling pathway originating in mitochondria modulates rat skeletal muscle membrane excitability. J Physiol. 2003;548(Pt 1):139-45.

17 Xu X, Zhao X, Liu TC, Pan H. Low-intensity laser irradiation improves the mitochondrial dysfunction of C2C12 induced by electrical stimulation. Photomed Laser Surg. 2008;26(3):197-202.

18 Silveira PC, Silva LA, Fraga DB, Freitas TP, Streck EL, Pinho R. Evaluation of mitochondrial respiratory chain activity in muscle healing by low-level laser therapy. J Photochem Photobiol B. 2009;95(1):89-92. 